
\title{
$\angle S$ Research Suare \\ Berberine Inhibits FoxM1 Dependent Transcriptional Regulation of POLE2 and Interferes with the Survival of Lung Adenocarcinoma
}

Lulu Ni

Jiangnan University

Ping Sun

Wuxi No 2 People's Hospital

Xiaochun Fan

Wuxi No 2 People's Hospital

Zhongjie Li

Jiangnan University

Hongli Ren

Shanghai University of Traditional Chinese Medicine

Jiangan Li ( $\nabla$ lijiangan2014@163.com )

Wuxi No 2 People's Hospital https://orcid.org/0000-0003-2203-6832

Research

Keywords: Berberine, FOXM1, POLE2, DNA replication, Lung adenocarcinoma, Survival.

Posted Date: September 14th, 2021

DOI: https://doi.org/10.21203/rs.3.rs-845748/v1

License: (1) This work is licensed under a Creative Commons Attribution 4.0 International License.

Read Full License

Version of Record: A version of this preprint was published at Frontiers in Pharmacology on January 31st, 2022. See the published version at https://doi.org/10.3389/fphar.2021.775514. 


\section{Abstract}

Background囚Berberine is one of the most interesting and promising natural anticancer drugs. POLE2 is involved in many cellular functions such as DNA replication and is highly expressed in a variety of cancers. However, the specific molecular mechanism of berberine interfering with POLE2 expression in lung adenocarcinoma (LUAD) is still unknown to a great extent.

Method:The KEGG database (Release 91.0) and Gene Ontology (GO) category database were used for functional annotation of differentially expressed genes after berberine treatment. Reproducibility assessment using TCGA dataset. The biological functions of berberine in LUAD were investigated by a series of in vitro and in vivo experiments: MTT, colony formation, mouse xenograft and plasmid transfection. The molecular mechanisms of berberine were demonstrated by plasmid transfection, quantitative RT-PCR and Western blotting.

Result:The elevated expression of FoxM1 and the high enrichment of DNA replication pathway were confirmed in LUAD by microarray and TCGA analysis, and were positively correlated with poor prognosis. Functionally, berberine inhibited the proliferation and survival of LUAD cell lines in vitro and in vivo. Mechanistically, berberine treatment down regulated the expression of FoxM1which closely related to survival, survival related genes in cell cycle and DNA replication pathway, and significantly down regulated the expression of survival related POLE2. Interestingly, we found that the transcription factor FoxM1 could act as a bridge between berberine and POLE2.

Conclusion: Berberine significantly inhibited LUAD progression via the FoxM1/POLE2, and FoxM1/POLE2 may act as a clinical prognostic factor and a therapeutic target for LUAD. Berberine may be used as a promising therapeutic candidate for LUAD patients.

\section{Introduction}

Lung cancer is the malignant tumor with the highest incidence and mortality in the world, and $85 \%$ of cases are NSCLC ${ }^{1}$. Lung adenocarcinoma is the most common subtype of NSCLC, and the five-year overall survival rate is less than $18 \%^{2}$. The initiation of DNA replication is the basic process of cell proliferation, and malignant proliferation is the basic feature of lung cancer cells. It is well known that the occurrence of lung adenocarcinoma is the result of imbalance of tumor suppressor genes or oncogenes, which will lead to uncontrolled proliferation of lung adenocarcinoma cells. In clinical, many mature chemotherapeutic drugs were designed to directly or indirectly inhibit DNA synthesis. Targeted DNA therapy was usually effective for the proliferation and survival of lung adenocarcinoma cells, such as cisplatin and carboplatin. They cross-linked purine bases in DNA, prevented DNA replication and repair of lung adenocarcinoma cells and promoted cell death. However, the side effects of these targeted drugs such as gastrointestinal, nephrotoxicity, ototoxicity and infection were very obvious. Therefore, it is very important and urgent to find high-efficiency and low toxicity anti lung adenocarcinoma drugs. 
Plant medicine is a rich source of new pharmacological active agents against diseases. Berberine is a low toxic natural plant alkaloid with broad spectrum of biological and pharmacological activities, including anti diabetes ${ }^{3}$, antidiarrheal ${ }^{4}$, anti-cancer ${ }^{5}$ and antibacterial ${ }^{6}$. At present, berberine has been evaluated in many clinical trials. Berberine has low toxicity to healthy cells and high cytotoxicity to cancer cells, which makes its research in anticancer become the most promising. Since the first study on the cytotoxicity in cancer cells of berberine in $1986^{7}$, its anticancer properties have been proved in many cancer cell studies ${ }^{8-12}$, which makes berberine one of the most interesting and promising natural anticancer drugs.

Although many studies had shown that berberine against lung adenocarcinoma mainly focused on different cell signaling pathways, including Sin3A/TOP2B ${ }^{13}, \mathrm{Bcl}-2$ / $\mathrm{Bax}^{14}, \mathrm{mTOR}^{15}$ and NF- $\mathrm{k} \mathrm{B} / \mathrm{COX}-2$, Akt / ERK ${ }^{16}$, miR-19a / TF / MAPK ${ }^{17}$, etc. It was not clear whether berberine could exert anticancer effect by interfering with DNA replication, the basic process of lung adenocarcinoma cell proliferation. In this study, we provided evidence that berberine inhibited DNA replication levels in lung adenocarcinoma cells and Lewis tumor xenograft mice. Berberine inhibited the proliferation of lung adenocarcinoma cells by interfering with the expression of POLE2 involved in DNA replication mediated by transcription factor FoxM1. Our study analyzed the mechanism of berberine inhibiting the proliferation of lung adenocarcinoma by using gene chip technology and TCGA, and correlated the changes of genome and transcriptome with cancer cell proliferation, DNA replication and overall survival.

\section{Materials And Methods Experiment reagents}

Berberine were purchased from Shanghai Tongtian Biotechnology Co., Ltd. (shanghai, China). Berberine were dissolved in DMSO and stored at $-20^{\circ} \mathrm{C}$. RPMI 1640 and Dulbeberberineo's modified eagle medium (DMEM) for culture were purchased from Gibco (Grand Island, NY, USA), fetal bovine serum (FBS) was from HyClone (South Logan, USA) and trypsin was from Gibco (Grand Island, NY, USA). Thiazoles [3-(4,5Dimethylthiazol-2-yl)-2,5-diphenyl-2H-tetrazolium Bromide, Methylthiazolyldiphenyl-tetrazolium bromide (MTT)] were purchased from Sigma (St. Louis, MO, USA). Propidium iodide『PI囚and FITC-Annexin V were both purchased from BD Biosciences (San Jose, CA). RNA extraction kit, PrimeScript RT MasterMix kit, and SYBR Premix Ex Taq kit were provided by Takara (Dalian, China). Antibodies against POLE2 was obtained from Santa Cruz (CA, USA). Antibodies against FOXM1 and actin as well as secondary antibodies were purchased from Cell Signaling Technology (Danvers, MA, USA).

\section{Microarray data analysis}

Slides were scanned by GeneChip ${ }^{\circledR}$ Scanner 3000 (Cat\#00-00212, Affymetrix, Santa Clara, CA, US) and Command Console Software 4.0 (Affymetrix, Santa Clara, CA, US) with default settings. Raw data were normalized by MAS 5.0 algorithm. Statistical significance of differential expression of probe sets 
between groups were detected by student $t$ test. Probe set with $p$-value $\leqq 0.05$ and absolute fold change $\geqq 1.5$ were considered as differential expressed. One gene keeps only one most statistical significant differential expressed probe set.

\section{Functional characterization of DEGs}

The KEGG database (Release 91.0) and Gene Ontology (GO) category database were used for functional annotation of differentially expressed genes. Enrichment analysis of $\mathrm{GO}$ categories was performed by $\mathrm{R}$ clusterProfiler ( $v$ 3.14.3) package, and enrichment analysis of pathways was tested upon hypergeometric distribution by R 'phyper' function. Those GO categories a FDR $<0.05$ were considered as significant enriched. While pathways with a p-value $<0.05$ were regared as enriched. Only those $\mathrm{GO}$ categories or pathways contains $\geqq 5$ DEGs were kept for further analysis.

\section{Reproducibility assessment using TCGA dataset}

The gene expression profiles data of 526 LUAD patients and 59 adjacent cancer samples and clinical characteristics of matched patients were obtained from the Cancer Genome Atlas (TCGA) data portal ${ }^{18}$. LUAD sequencing data were downloaded. Differential expression of genes between patients and adjacent were detected by 'edgeR' ( $v$ 3.28.1) package, with a threshold of a FDR $\leqq 0.05$. Then functional enrichment analysis were performed on these DEGs as described above. The clinical data of 522 patients were used for Cox regression analysis.

\section{Cell culture and plasmid transfection}

Human NSCLC cell lines, A549, H1299, and H1975 cells were purchased from Shanghai Cell Bank of the Chinese Academy of Sciences. A549 cells were cultured with DMEM medium and H1299 and H1975 cells were grown in RPMI 1640 medium. Both DMEM medium and RPMI 1640 medium were supplemented with $10 \%$ fetal bovine serum, penicillin $(100 \mathrm{U} / \mathrm{ml})$ and streptomycin sulfate $(100 \mu \mathrm{g} / \mathrm{ml})$. Cells were maintained at $37^{\circ} \mathrm{C}$ in a humidified $5 \% \mathrm{CO} 2$ atmosphere. The empty plasmid EX-NEG-M02 (Genecopoeia) and human FoxM1-overexpression plasmid (Genecopoeia) were transfected into A549, H1299, and H1975 cells using Lipofectamine 3000 reagent (Invitrogen \#2024201, MA, USA), according to the manufacturer's instructions. After that, the A549, H1299, and H1975 cells transfected with the plasmid were used in subsequent experiments.

\section{MTT colorimetric analysis for determining inhibition of cell proliferation}


To assess the effect of berberine on the survival and proliferation of NSCLC cells, MTT analysis was adopted. A549, H1299 and $\mathrm{H} 1975$ cells in the logarithmic phase were seeded in a 96-well plate, at a density of 3000 cells/well and maintained at $37^{\circ} \mathrm{C}$ in a humidified $5 \% \mathrm{CO} 2$ atmosphere for $16 \mathrm{~h}$. Then the berberine at a density of $0,30,60,90,120,150,180,210,240,270 \mu \mathrm{M}$ were added, respectively. The total volume was $200 \mu \mathrm{l}$. Every concentration was set as well with 4 parallel wells in each group and kept at $37^{\circ} \mathrm{C}$ in a humidified $5 \% \mathrm{CO} 2$ atmosphere for 24,48 and 72 hours, respectively. When the time was due, $20 \mu \mathrm{l}$ of MTT reagent (Sigma) was added to each well in the 96-well plate. Then the plate was kept at $37^{\circ} \mathrm{C}$ for 4 hours. After removing the supernatants, $150 \mu \mathrm{l}$ of DMSO was supplemented into each well and then the plate was maintained at $37^{\circ} \mathrm{C}$ for 15 minutes. Absorbance $(A)$ was determined with the microplate reader at $570 \mathrm{~nm}$. The cell inhibition was calculated as follows:

Inhibition rate $(\%)=[(A$ of negative control group $-A$ of test group $) / A$ of negative control group $] \times 100 \%$

\section{Determination of sphere formation efficiency}

To clarify the effect of berberine on the tumorigenesis ability of NSCLC cells, plate clone assay was applied. A549, H1975 and H1299 cells (2000 for each type) in the logarithmic phase were seeded in a 6well plate for 16 hours. Then berberine at a density of $0,5,10$ and $20 \mu \mathrm{M}$ were added to NSCLC cells, respectively. The cells were maintained at $37^{\circ} \mathrm{C}$ in a humidified $5 \% \mathrm{CO} 2$ atmosphere. Ten days later, the colonies were stained with crystal violet and photographs of the stained colonies were taken by the digital camera and dissecting microscope.

\section{Determination of the effects on transplanted tumor in C57BL/6 mice}

C57BL/6 mice aged at 6-8 weeks and weighed at 18-22 $\mathrm{g}$ were purchased from SLAC Shanghai and fed in a standard feeding atmosphere at Jiangnan University. LLC cells $(3 \times 106)$ were suspended in the medium at $150 \mu \mathrm{l}$ and then subcutaneously injected into the right axilla of C57BL/ 6 mice. After LLC cells were injected, berberine groups were given an intraperitoneal injection of berberine (100, 200 and 400 $\mathrm{mg} / \mathrm{Kg}$ ) continually for 4 weeks. The blank control group was administered intraperitoneally injected with PBS. The volume of the tumor was measured every three days with a vernier caliper. The formula for volume was as follows: $V=\llbracket \Pi / 8 \rrbracket \times a \times b 2$. "a" represents the maximum diameter of the tumor, while " $b$ " reflects the shorter diameter vertical to "a". The C57BL/ 6 mice with the tumor over $2000 \mathrm{~mm} 3$ was put to death by $\mathrm{CO} 2$ suffocation.

\section{HE staining of mouse tumor were observed}

The tumors of mice were taken and soaked in $4 \%$ paraformaldehyde solution. The $4 \%$ paraformaldehyde solution was changed every day. One week later, the fixed specimens were routinely dehydrated with 
alcohol gradient, transparent xylene, embedded with paraffin, and sectioned $5 \mathrm{~m}$ thick. The tumor sections were stained with $\mathrm{HE}$ and were observed under microscope.

\section{Immunohistochemistry Analysis}

To demonstrate the expression of FOXM, immunohistochemistry was used to detect. The tumor sections of mice in each group were incubated with FOXM1 (Cell Signaling Technology, MA, USA) and detected, respectively, with the secondary antibodies(Cell Signaling Technology, MA, USA).

\section{Quantitative RT-PCR}

Total RNA from A549, $\mathrm{H} 1299$ and $\mathrm{H} 1975$ cells was extracted using Trizol reagent after the treatment of 0 , $30,60,120 \mu \mathrm{M}$ berberine for $24 \mathrm{~h}$. $1 \mu \mathrm{g}$ of total RNA was transcribed to cDNA using the PrimeScriptTM RT Master Mix kit (TaKaRa, China) aberberineording to the protocols. Quantitative RT-PCR was performed in a reaction volume of $20 \mu \mathrm{L}$ cDNA on ABI system (Applied Biosystems, Life Technologies), and carried out with the following parameters: $95^{\circ} \mathrm{C}$ for $30 \mathrm{~s}$, amplifications were carried out with 40 cycles at a melting temperature of $95^{\circ} \mathrm{C}$ for $5 \mathrm{~s}$ and an annealing temperature of $60^{\circ} \mathrm{C}$ for $30 \mathrm{~s}$, followed by melt curve analysis. The relative expression was calculated using the 2- $\triangle \triangle \mathrm{Ct}$. The following 4 genes were selected for analysis: RRM1, RRM2, POLE2, and LIG1. An 18S was used as an internal reference gene to normalize the expression of all genes. Primers for all genes were listed as follows: $18 \mathrm{~S}$ forward:

GTAACCCGTTGAACCCCATT,

18 S reverse: CCATCCAATCGGTAGTAGCG.

POLD1 forward: ATCCAGAACTTCGACCTTCCG,

POLD1 reverse: ACGGCATTGAGCGTGTAGG.

DNA2 forward: AGAGCTGTCCTGAGTGAAACT,

DNA2 reverse: GAAACACCTCATGGAGAACCG.

POLE2 forward: TTTTGCAGAAGTCTTCACAGATG,

POLE2 reverse: GCAGAAGGTTGGTTTGAAGA.

RFC5 forward: GAAGCAGACGCCATGACTCAG,

RFC5 reverse: GACCGAACCGAAACCTCGT.

MCM2 forward: ATCTACGCCAAGGAGAGGGT,

MCM2 reverse: GTAATGGGGATGCTGCCTGT. 
MCM4 forward: CTGTCCATTGCAAAGGCTG,

MCM4 reverse: GAGACTCAATGGGATTTGCTG.

MCM6 forward: CTGTGATGAGGTCCAACCT,

MCM6 reverse: GACATCAGGTGTTTCCACAC.

RFC3 forward: CCCTGAGACAGATTGGGAG,

RFC3 reverse: TCAAGGAGCCTTTGTGGAG.

FEN1 forward: GATGATTTCTTCAAGCCTTGAC,

FEN1 reverse: TCACAAACACAGACACAGC.

POLA2 forward: CTCTCCAAGTGCTACTCCC,

POLA2 reverse: ATACTCCCTGTGCTAAGCC.

PRIM1 forward: CTTAAACTTTATTACCGGAGGC,

PRIM1 reverse: GTAATTCTTTATCACTCCACCG.

PRIM2 forward: GGTTTAACTTTGGAACAGGC,

PRIM2 reverse: TTCCTCTTCCTTGTCTGGA.

\section{Western blotting for determining protein}

A549, H1299 and H1975 cells in the logarithmic phase were seeded in $10 \mathrm{~cm}$ culture dishes. When cells occupied $80 \%$ of the dish, berberine were used for intervention. berberine at a density of $0,30,60,120 \mu \mathrm{M}$ were added to A549, H1299 and H1975 cells, respectively. 24 hours later, $100 \mu$ RIPA was added to the dishes and cells were fully soaked. After centrifugation for 30 minutes, supernatants were collected for protein measurement. After being washed with $95^{\circ} \mathrm{C}$ water for 10 minutes, the proteins were separated with gel and transferred to a membrane. The membranes were incubated overnight at $4^{\circ} \mathrm{C}$ with the primary antibodies and then further incubated with secondary antibodies and finally visualized.

\section{Statistical analysis}

The relationship between cell cycle or DNA repair pathway related gene expression and overall survival of TCGA - LUAD patients was analyzed using COX regression. $P<0.05$ was considered to have significant statistical significance. all these analysis were conducted using $R$ (v. 3.6.0). 


\section{Results}

\section{Screening of differentially expressed genes in microarray}

To screen for potential differentially expressed genes, we analyzed samples from berberine treated NSCLC. The results showed that most genes were down regulated after berberine treatment, compared with control group (Figure 1A), and the differential expressed genes after berberine treatment were mainly enriched in cell cycle and DNA replication pathway, and tended to be down regulated (Figure 1B). Next, we investigate the importance of cell cycle and DNA replication related genes in the network. We analyzed whether the interaction strength of cell cycle and DNA replication related genes with other differential genes screened from the whole drug data was significantly different from that of random network, and investigated two indicators: interaction number and LCC. We found that the PPI interaction number was significantly higher than that of the random network (Figure 1C), and the LCC was higher than that of the random network (Figure 1D), both of which met the pvalue $<0.05$. From the results, the cell cycle and DNA replication genes in the network diagram were more important in the differential genes after drug action. In addition, compared with the control group, 228 genes were up-regulated, 737 genes were down regulated, and 105 genes related to cell cycle and DNA replication were significantly down regulated after berberine treatment (Figure 1E). Go enrichment analysis also showed that these differentially expressed genes were mainly enriched in cell cycle and DNA replication (Figure 1F).

\section{Berberine down regulated survival related genes in cell cycle and DNA replication pathway}

To further explore the effect of berberine on cell cycle and DNA replication, we analyzed the data in 59 adjacent normal tissues and 526 Lung adenocarcinoma (LUAD) tumor tissues from The Cancer Genome Atlas (TCGA). 8876 genes were down regulated and 4937 genes were up-regulated when adjacent normal tissues compared with the tumor by the cutoff of a FDR<0.05. Functional enrichment of these DEGs showed that Cell cycle pathway was statistically enriched and up-regulated in tumor. Compare with 646 DEGs from berberine treated lung adenocarcinoma cells, 480 genes were common differential expressed, among which 321 were common up and 56 common down regulated. Functional enrichment of these 377 coexist DEGs with the same up-down direction showed that cell cycle and DNA replication pathways were the most significant enriched (Figure 2A). And berberine could effectively down regulate cell cycle and DNA replication (Figure 2B and $2 \mathrm{C}$ ). In TCGA, the differential genes were mainly enriched in purine metabolism and cell cycle, and tended to be up-regulated. But, almost all of the differentially expressed genes in DNA replication were up-regulated (Figure supplement 1). All these results suggested that berberine might interfere with tumor progression mainly by down regulating gene expression of cell cycle and DNA replication.

Next, we wondered whether berberine would selectively act on survival related genes, rather than all genes in cell cycle and DNA replication. Firstly, we classify these genes into survival or non-survival realated by 
Cox ph analyasis. Genes with a p-value $<0.05$ were regarded as survival related. We found that survival related genes tend to be differentially expressed genes, and most of them were down regulated (Figure 2D), especially reflected in DNA replication pathway. Nearly $90 \%$ survival related genes in DNA replication were DEGs and all of them were down regulated (Figure 2E), and berberine also had more significant effect on differential survival related genes in DNA replication pathway (Figure 2F). In addition, we also found that there were some survival related genes in cell cycle or DNA replication pathway in TCGA analysis. Although there was no significant difference after berberine treatment, a large proportion tended to be down regulated (Fig. supplementary 2).

\section{Berberine down regulated the expression of FoxM1 related to survival}

To further investigate the effect of berberine on survival related genes in DNA replication pathway, then we analyzed the transcriptional regulation of DNA replication related differential genes after berberine treatment. The results showed that except FOSL1, the numbers of target genes regulated by other transcription factors or their cofactors were almost the same (Figure 3A). Next, we carried out the survival analysis of the above transcription factors and their cofactors in TCGA data, and only FoxM1 met the pvalue $<0.05$, showed high expression and low survival (Figure 3B). After that, we verified it on non-small cell lung cancer cells. The results showed that the expression of FoxM1 was down regulated in A549, $\mathrm{H} 1299$ and $\mathrm{H} 1975$ cells after berberine treatment, and it was the most significant in H1975 cells (Figure $3 \mathrm{C}$ and $3 \mathrm{D})$.

\section{Berberine inhibited the survival of NSCLC cells}

Since berberine could inhibit the expression of FoxM1 related to survival, whether berberine could really inhibit the survival of NSCLC cells, then we carried out the cellular verification. A549, H1299 and H1975 cells were treated with berberine $(0,30,60,90,120,150,180,210,240,270 \mu \mathrm{M})$ for $24 \mathrm{~h}, 48 \mathrm{~h}$ and $72 \mathrm{~h}$, respectively. The results showed that when the concentration was $120 \mu \mathrm{M}$, the number of NSCLC cells was reduced and the morphology shrunked significantly (Figure 4A and 4B), and the inhibitory effect was enhanced with the increase of concentration (Figure 4B). Berberine is quite sensitive to H1975 cells, even at very low concentration, the inhibition rate was very high (Figure 4B). Then, we further studied the effect of berberine on the tumorigenesis of NSCLC cells by plate cloning. The results showed that after treatment of berberine $(0,5,10,20 \mu \mathrm{M})$, the clone number of NSCLC cells decreased with the increase of concentration (Figure 4C and 4D), 10 and $20 \mu \mathrm{M}$ could completely inhibit the formation of NSCLC cells ( $P$ $<0.001, P<0.001, P<0.001$ ) (Figure 4D). At high magnification, we found that the clone size of control group was very large, while that of berberine group was significantly reduced (Figure $4 C$ ). These results suggest that berberine could inhibit the survival of NSCLC cells. 


\section{Berberine inhibited the survival of lung cancer xenografts and down regulated the expression of FoxM1 in vivo}

In lung cancer cells, we have just confirmed that berberine can inhibit the survival of NSCLC cells. Then we tested the effect of berberine on the survival of lung cancer xenografts in more complex animals. We injected LLC cells into the right armpit of c57bl/6 mice. Berberine with 100, 200 and 400mg/kg concentration were used for gastric administration. After 4 weeks, we found that high, medium and low concentrations of berberine could significantly inhibit the growth of lung cancer transplantation $(P<0.01$, $P<0.001, P<0.001$ ) (Figure 5A and 5B), and the medium concentration of berberine had the most significant inhibition effect on the growth of lung cancer transplantation (Figure 5A and 5B). In addition, berberine with medium and low concentration significantly prolonged the survival time of lung cancer transplanted mice (Figure $5 \mathrm{C}$ ), and there was no significant difference in weight between the mice in each group (Figure 5D). Then we performed HE staining and immunohistochemistry analysis of the tumor. The results of microscope showed that the tumor necrosis area of berberine group was significantly increased compared with the blank control group (Figure $5 \mathrm{E}$ ). And berberine could significantly reduce the expression of FoxM1 in tumor $(P<0.05)$ (Figure 5F).

\section{Berberine interfered the expression of survival related genes POLE2 in DNA replication mediated by FoxM1}

To verify that berberine inhibited the survival related genes in DNA replication pathway through FoxM1. We further analyzed the network relationship between FoxM1 and DNA replication related differential genes after berberine treatment, and found that not all the differentially expressed target genes were survival related (Figure 6A). Then, we used univariable Cox proportional hazards regression analysis to analyze the survival of DNA replication related genes in TCGA data, and found out the survival related genes in DNA replication (Figure 6B). We verified the common genes of the above two analyses by PCR in A549 cells and found that berberine down regulated the expression of MCM4, POLA2, POLE2 and PRIM1 in A549 cells (Figure 6C). At the same time, we found that MCM4, POLA2, POLE2 and PRIM1 were involved in G1/S transition of mitotic cell cycle and DNA replication, while berberine could significantly down regulate their expression, especially on POLE2 and PRIM1 (Figure 6D). And berberine could significantly down regulate POLE2 and PRIM1 in A549 cells, H1299 cells and H1975 cells (Figure 6E). In addition, we found that berberine did not reduce the expression of POLE2 when FoxM1 was overexpressed, but could significantly reduce the expression of PRIM1 (Figure 6F), indicating that berberine affected the survival of NSCLC cells by inhibiting the expression of POLE2 through FoxM1.

\section{Discussion}

Gene chip technology and Cancer Genome Atlas (TCGA) have been proved to be reliable diagnostic and prognostic tools for cancer patients ${ }^{19-21}$. This independent data stored in a public database enables 
researchers to explore the potential mechanisms of diagnosis and treatment. In this study, in order to reveal the molecular mechanism of berberine intervention in the survival of lung adenocarcinoma cells, we analyzed the differential expression of genes in A549 cells treated with berberine and in the lung adenocarcinoma cohort created by TCGA, then determined the importance of DNA replication pathway, and deeply analyzed the relationship between DNA replication of lung adenocarcinoma cells and berberine intervention in the survival of lung adenocarcinoma patients. The results showed that the differentially expressed genes were mainly enriched in the cell cycle and DNA replication pathway after berberine treatment, and were significantly down regulated. We used the samples of lung adenocarcinoma tumor and adjacent tissues in cancer genome map and lung adenocarcinoma cells before and after berberine treatment for common differential expressed gene analysis. The results showed that there were $321+56$ common differentially expressed genes, mainly related to cell cycle and DNA replication pathway. After berberine treatment, these differentially expressed genes were mainly downregulated, and most of these differentially expressed genes were survival related genes. Berberine had a more significant effect on differential survival related genes in DNA replication pathway than that in cell cycle pathway. These results suggested that berberine could significantly affect the survival related genes in lung adenocarcinoma, especially in the DNA replication pathway.

It is well known that transcription factors (TF) play a major role in tumorigenesis and tumor progression by widely promoting or blocking the transcription of their targets. Identifying TF with malignant characteristics can provide a comprehensive view for explaining tumor biology. Forkhead / wing helix domain transcription factor FoxM1, as an oncoprotein, affected the occurrence and development of cancer through trans activation of related oncogenes ${ }^{22,23}$. Its expression had been proved to be increased in various cancers ${ }^{22,24}$. It was worth noting that FoxM1 had been reported as the primary gene expression biomarker with poor prognosis in Pan-cancer analysis, which includes $>18,000$ tumors from 39 different malignancies ${ }^{25}$. The high expression of FoxM1 was closely related to the reduction of patient survival $^{26,27}$. People were interested in the therapeutic target of FoxM1 in cancer ${ }^{28,29}$. FoxM1 had recently been identified as a key transcriptional regulator of related oncogenes in lung adenocarcinoma. In our study, the expression of FoxM1 was down regulated in lung adenocarcinoma cells after berberine treatment, most significantly in $\mathrm{H} 1975$ cells. At the same time, berberine could also inhibit the growth and clone formation of lung adenocarcinoma cells. In vivo, we also found that berberine inhibited the survival of lung adenocarcinoma xenografts and significantly down regulated the expression of FoxM1. In conclusion, berberine could significantly inhibit the survival of lung adenocarcinoma through FoxM1.

FoxM1 played an important role in DNA replication. FoxM1 was recently reported to induce DNA replication pressure in vitro, and FoxM1 expression was observed to be associated with the expression of DNA replication pressure biomarkers in several cancer types ${ }^{30}$. In order to evaluate the role of FoxM1 in DNA replication of lung adenocarcinoma cells, we further analyzed the transcriptional regulation of related differential genes in DNA replication pathway. We found that the number of target genes regulated by transcription factors or their cofactors was almost the same except FOSL1. However, in TCGA data, we analyzed the survival of the above transcription factors and their cofactors, and only FoxM1 met $\mathrm{P}<0.05$,

Page $11 / 23$ 
showing high expression and low survival rate. We further analyzed the network relationship between FoxM1 and related differentially expressed genes in DNA replication pathway after berberine treatment, and also found that most differentially expressed target genes were related to survival. We used univariate Cox proportional hazards regression analysis to find survival related genes in DNA replication in TCGA data. Then, the common differential expressed gene in the above two analyses was verified by PCR. It was found that berberine could down regulated the expression of POLE2 and PRIM1.

It was worth noting that our study also found FoxM1 was closely related to POLE2. POLE2 was involved in cell functions, such as DNA replication, repair and cell cycle control ${ }^{31}$, as well as array based proliferation characteristics ${ }^{32}$. On the other hand, POLE2 had been previously reported to be highly expressed in breast cancer, colorectal cancer, cervical cancer and bladder cancer ${ }^{33-35}$. We overexpressed FoxM1 and found that berberine could not reduce the expression of POLE2, but could significantly reduce the expression of PRIM1, indicating that FoxM1 mediated the expression of POLE2. In conclusion, berberine intervened the survival of lung adenocarcinoma cells by inhibiting the expression of POLE2 mediated by FoxM1.

In conclusion, we found that berberine could significantly inhibit the DNA replication pathway in lung adenocarcinoma cells through gene chip technology of lung adenocarcinoma A549 cells and TCGA data analysis. We found that berberine could significantly inhibit the proliferation of lung adenocarcinoma cells in vitro and in vivo. In addition, we demonstrated that the therapeutic target of berberine was to inhibit the expression of FoxM1 and POLE2 mediated by FoxM1, so as to intervene the survival of lung adenocarcinoma (General block diagram). This is the first study on the mechanism of berberine in the treatment of lung adenocarcinoma by regulating FoxM1 and POLE2 mediated by FoxM1. It is also the first time to confirm the significance and relationship of FoxM1 and its target gene POLE2 in lung adenocarcinoma.

\section{Abbreviations}

NSCLC: Non small cell lung cancer

LUAD: lung adenocarcinoma

POLE2: DNA Polymerase Epsilon 2

FoxM1: Forkhead Box M1

GO: Gene Ontology

TCGA: The Cancer Genome Atlas

Bcl-2: B-cell lymphoma-2

TF: Transcription factors 
NF-kB: nuclear factor-kappa B

COX-2: Cyclooxygenase 2

miR-19a: microRNA-19a

MAPK: mitogen-activated protein kinase

Akt: Protein kinase B

ERK: extracellular regulated protein kinases

PRIM1: DNA Primase Subunit 1

\section{Declarations}

Ethics declarations

All of the procedures were reviewed and approved by the Animal Ethics Committee of Jiangnan University before and during the experiment.

Consent to publish

Not applicable.

Availability of data and materials

The datasets used and analysed during the current study are available from the corresponding author on reasonable request.

Competing interests

The authors declare that they have no competing interests.

Funding

This study was supported by National Natural Science Foundation of China (81904171), Jiangsu Postdoctoral Research Foundation (2020Z388), Top Talent Support Program for young and middle-aged people of Wuxi Health Committee, General fund of Wuxi health committee (M202033).

Author information

Affiliations

\section{Department of Basic Medicine, Jiangnan University, Wuxi, 214122, China}

Lulu Ni, Zhongjie Li 
Department of emergency, the Affiliated Wuxi NO.2 People's Hospital of Nanjing Medical University, Wuxi, 214000, China

Jiangan Li, Xiaochun Fan

Institute of Science, Technology and Humanities, Shanghai University of Traditional Chinese Medicine, Shanghai 201203, China

Hongli Ren

Department of pathology, the Affiliated Wuxi NO.2 People's Hospital of Nanjing Medical University, Wuxi 214000, China

Ping Sun

Contributions

LN and JL conceived and designed the study, LN, PS and XF performed the experiments and collected the data, ZL and HR contributed to analyzing the data, NL wrote the manuscript, JL and HR made manuscript revisions. All authors have read and approved the manuscript.

Corresponding authors

Correspondence to Jiangan Li or Hongli Ren.

Acknowledgements

Not applicable.

\section{References}

1. Torre LA, Bray F, Siegel RL, et al. Global cancer statistics, 2012. CA Cancer J Clin. 2015,65(2):87-108.

2. Siegel RL, Miller KD, Jemal A. Cancer statistics, 2019. CA Cancer J Clin. 2019,69(1):7-34.

3. Wang JT, Peng JG, Zhang JQ, et al. Novel berberine-based derivatives with potent hypoglycemic activity. Bioorg Med Chem Lett. 2019,29(23):126709.

4. Joshi PV, Shirkhedkar AA, Prakash K, et al. Antidiarrheal activity, chemical and toxicity profile of Berberis aristata. Pharm Biol. 2011,49(1):94-100.

5. Liu D, Meng X, Wu D, et al. A Natural Isoquinoline Alkaloid With Antitumor Activity: Studies of the Biological Activities of Berberine. Front Pharmacol. 2019,10:9.

6. Peng L, Kang S, Yin Z, et al. Antibacterial activity and mechanism of berberine against Streptococcus agalactiae. Int J Clin Exp Pathol. 2015,8(5):5217-5223.

7. Wang MX, Huo LM, Yang HC, et al. An experimental study on the photodynamic activity of berberine in vitro on cancer cells. J Tradit Chin Med. 1986,6(2):125-127. 
8. Och A, Zalewski D, Komsta L, et al. Cytotoxic and Proapoptotic Activity of Sanguinarine, Berberine, and Extracts of Chelidonium majus L. and Berberis thunbergii DC. toward Hematopoietic Cancer Cell Lines. Toxins (Basel). 2019,11(9).

9. Kettmann V, Kosfalova D, Jantova S, et al. In vitro cytotoxicity of berberine against HeLa and L1210 cancer cell lines. Pharmazie. 2004,59(7):548-551.

10. Yu FS, Yang JS, Lin HJ, et al. Berberine inhibits WEHI-3 leukemia cells in vivo. In Vivo. 2007,21(2):407-412.

11. Pazhang $Y$, Ahmadian $S$, Mahmoudian $M$, et al. Berberine-induced apoptosis via decreasing the survivin protein in K562 cell line. Med Oncol. 2011,28(4):1577-1583.

12. Zheng F, Tang Q, Wu J, et al. p38alpha MAPK-mediated induction and interaction of FOXO3a and p53 contribute to the inhibited-growth and induced-apoptosis of human lung adenocarcinoma cells by berberine. J Exp Clin Cancer Res. 2014,33:36.

13. Chen J, Huang $X$, Tao $C$, et al. Berberine chloride suppresses non-small cell lung cancer by deregulating Sin3A/TOP2B pathway in vitro and in vivo. Cancer Chemother Pharmacol. 2020,86(1):151-161.

14. Li J, Liu F, Jiang S, et al. Berberine hydrochloride inhibits cell proliferation and promotes apoptosis of non-small cell lung cancer via the suppression of the MMP2 and Bcl-2/Bax signaling pathways. Oncol Lett. 2018,15(5):7409-7414.

15. Kumar R, Awasthi M, Sharma A, et al. Berberine induces dose-dependent quiescence and apoptosis in A549 cancer cells by modulating cell cyclins and inflammation independent of mTOR pathway. Life Sci. 2020,244:117346.

16. Lu JJ, Fu L, Tang Z, et al. Melatonin inhibits AP-2beta/hTERT, NF-kappaB/COX-2 and Akt/ERK and activates caspase/Cyto $C$ signaling to enhance the antitumor activity of berberine in lung cancer cells. Oncotarget. 2016,7(3):2985-3001.

17. Chen QQ, Shi JM, Ding Z, et al. Berberine induces apoptosis in non-small-cell lung cancer cells by upregulating miR-19a targeting tissue factor. Cancer Manag Res. 2019,11:9005-9015.

18. Liu J, Lichtenberg T, Hoadley KA, et al. An Integrated TCGA Pan-Cancer Clinical Data Resource to Drive High-Quality Survival Outcome Analytics. Cell. 2018,173(2):400-416 e411.

19. Team AS. Shared Gene Expression Alterations in Nasal and Bronchial Epithelium for Lung Cancer Detection. J Natl Cancer Inst. 2017,109(7).

20. Sato T, Kaneda A, Tsuji S, et al. PRC2 overexpression and PRC2-target gene repression relating to poorer prognosis in small cell lung cancer. Sci Rep. 2013,3:1911.

21. Wang $Q$, Gan $H$, Chen $C$, et al. Identification and validation of a 44-gene expression signature for the classification of renal cell carcinomas. J Exp Clin Cancer Res. 2017,36(1):176.

22. Halasi M, Gartel AL. FOX(M1) news-it is cancer. Mol Cancer Ther. 2013,12(3):245-254.

23. Kalathil D, John S, Nair AS. FOXM1 and Cancer: Faulty Cellular Signaling Derails Homeostasis. Front Oncol. 2020,10:626836. 
24. Nandi D, Cheema PS, Jaiswal N, et al. FoxM1: Repurposing an oncogene as a biomarker. Semin Cancer Biol. 2018,52(Pt 1):74-84.

25. Gentles AJ, Newman AM, Liu CL, et al. The prognostic landscape of genes and infiltrating immune cells across human cancers. Nat Med. 2015,21(8):938-945.

26. Li M, Sun Q, Wang X. Transcriptional landscape of human cancers. Oncotarget. 2017,8(21):3453434551.

27. Barger CJ, Branick C, Chee L, et al. Pan-Cancer Analyses Reveal Genomic Features of FOXM1 Overexpression in Cancer. Cancers (Basel). 2019,11(2).

28. Tabatabaei Dakhili SA, Perez DJ, Gopal K, et al. A structure-activity relationship study of Forkhead Domain Inhibitors (FDI): The importance of halogen binding interactions. Bioorg Chem. 2019,93:103269.

29. Ziegler Y, Laws MJ, Sanabria Guillen V, et al. Suppression of FOXM1 activities and breast cancer growth in vitro and in vivo by a new class of compounds. NPJ Breast Cancer. 2019,5:45.

30. Li Z, Yu DS, Doetsch PW, et al. Replication stress and FOXM1 drive radiation induced genomic instability and cell transformation. PLoS One. 2020,15(11):e0235998.

31. Burgers PM. Eukaryotic DNA polymerases in DNA replication and DNA repair. Chromosoma. 1998,107(4):218-227.

32. Rosenwald $A$, Wright $G$, Wiestner $A$, et al. The proliferation gene expression signature is a quantitative integrator of oncogenic events that predicts survival in mantle cell lymphoma. Cancer Cell. 2003,3(2):185-197.

33. Zhou Q, Effati R, Talvinen K, et al. Genomic changes of the $55 \mathrm{kDa}$ subunit of DNA polymerase epsilon in human breast cancer. Cancer Genomics Proteomics. 2008,5(5):287-292.

34. Chubb D, Broderick P, Dobbins SE, et al. Rare disruptive mutations and their contribution to the heritable risk of colorectal cancer. Nat Commun. 2016,7:11883.

35. Liu D, Zhang XX, Xi BX, et al. Sine oculis homeobox homolog 1 promotes DNA replication and cell proliferation in cervical cancer. Int J Oncol. 2014,45(3):1232-1240.

\section{Figures}



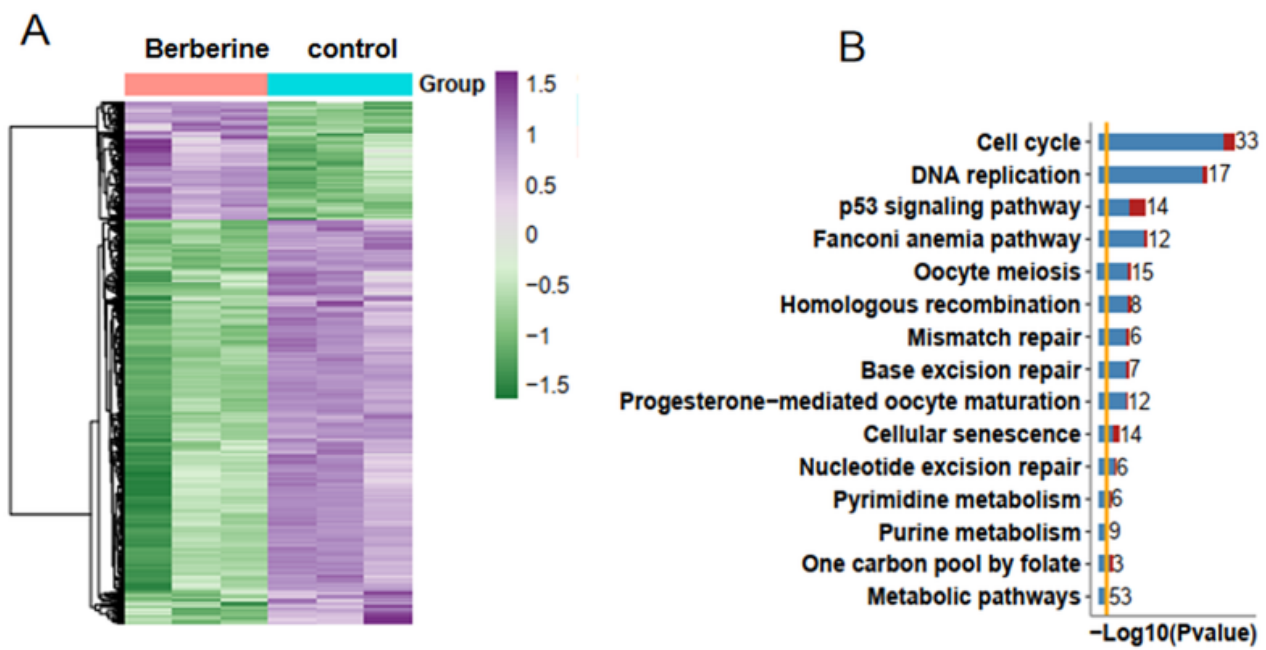
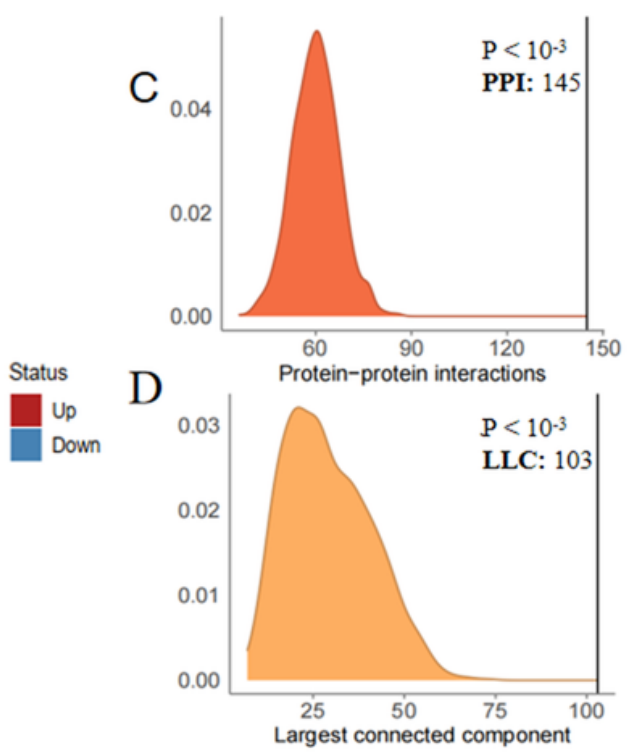

E

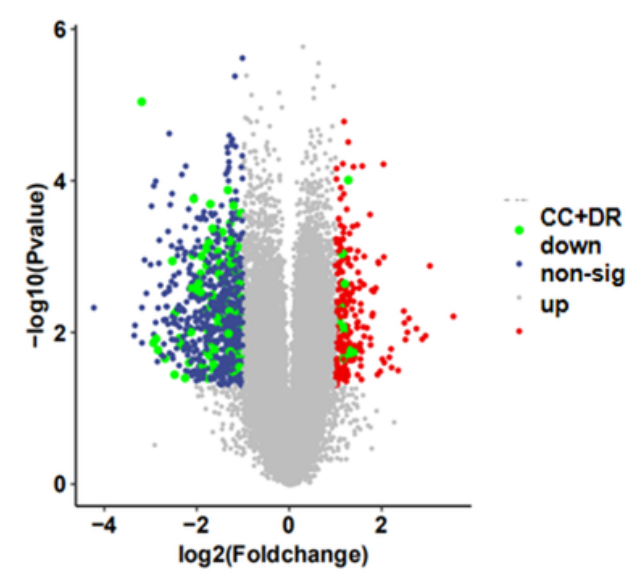

$\mathrm{F}$

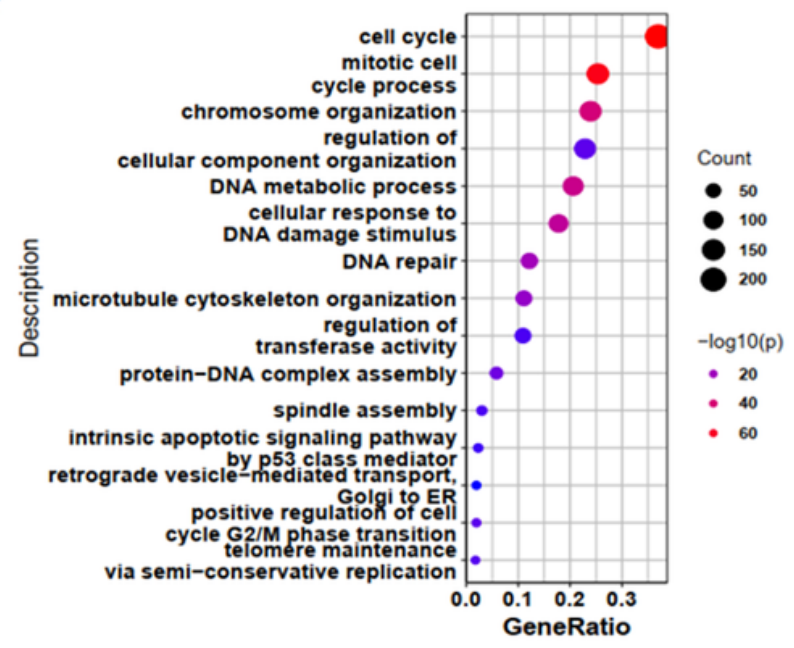

Figure 1

Potential antitumour mechanisms of berberine in NSCLC. (A) Differential gene thermography showed that the gene expression in human A549 cells had a good consistency, and the gene expression in Berberine treatment group was low. (B) KEGG enrichment analysis showed that the differential genes were mainly enriched in cell cycle and DNA replication pathway after berberine treatment, and tended to be down regulated. (C) Number of protein-protein interactions (PPIs) in the network, revealing significant enrichment for PPIs between Cell cycle and DNA replication genes relative to random expectation $(p<10-$ 3). (D) Size of the largest connected component (LCC) in the network revealing cell cycle and DNA replication genes occupy a distinct region of the differential genes $(p<10-3)$. (E) Differential probe volcanogram showed that cell cycle and DNA replication related genes were significantly down regulated. (F) Go enrichment analysis showed that the differential genes after berberine treatment were mainly enriched in cell cycle and DNA replication. 


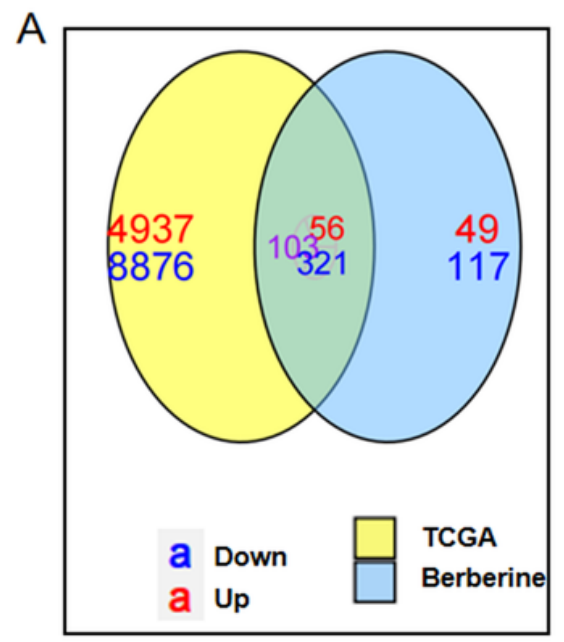

$\mathrm{D}$

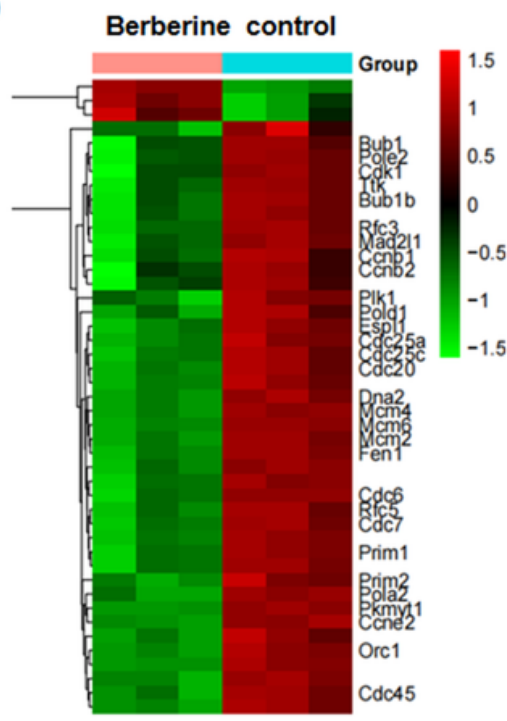

B

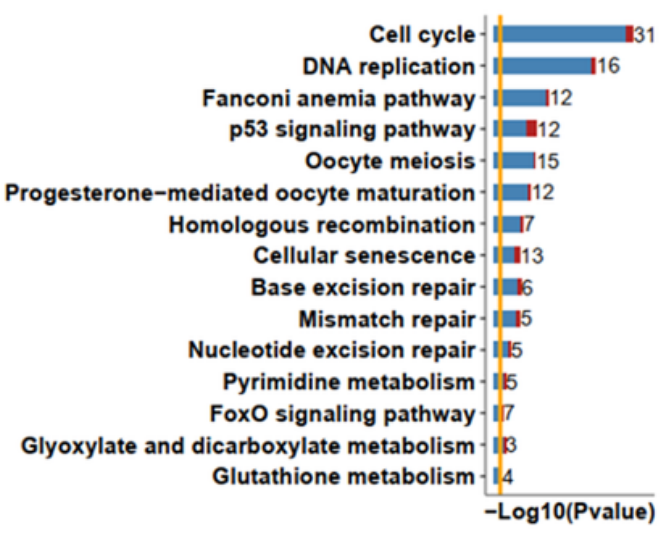

E

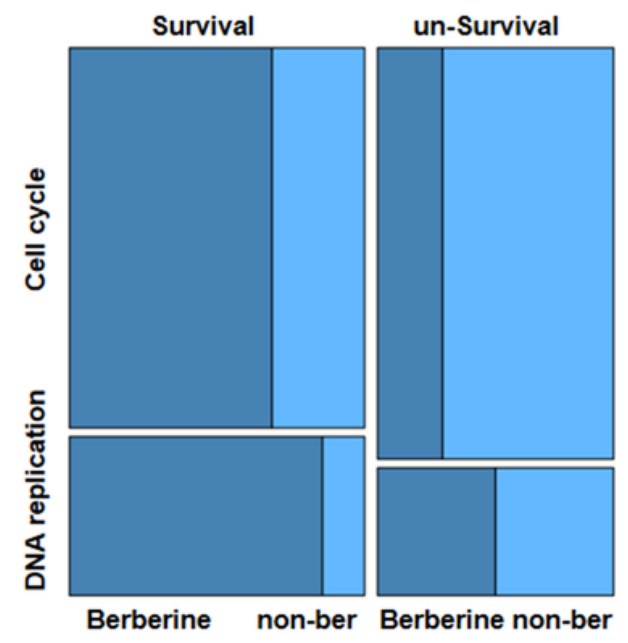

C

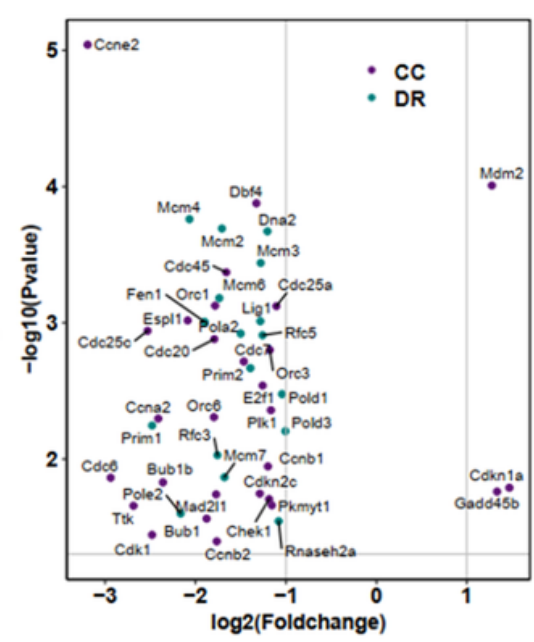

F

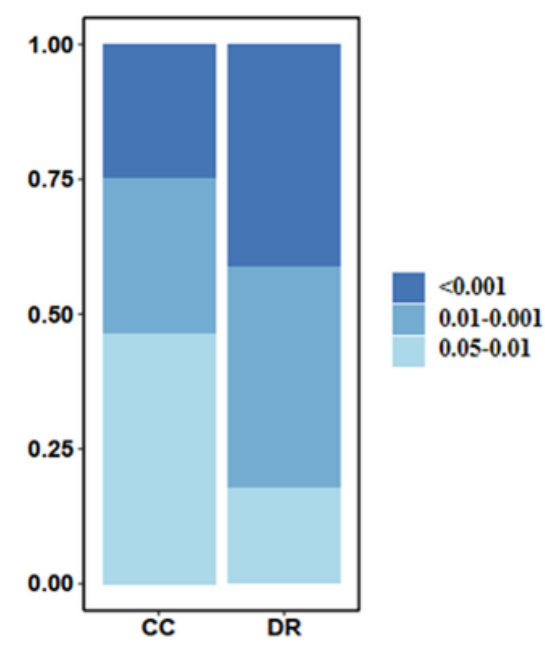

Figure 2

Berberine down regulates survival related genes in DNA replication pathway. (A)The Venn map of differential genes (compared with TCGA) showed that berberine could change the up-regulated genes into down-regulated genes, and the enrichment of $(321+56)$ genes with the same up and down-regulated direction after berberine treatment and in TCGA were mainly related to cell cycle and DNA replication. (B)KEGG enrichment analysis showed that the common differential genes in berberine treatment and TCGA were mainly enriched in cell cycle and DNA replication pathway, and tended to be down regulated.

(C)Scatter plot showed that cell cycle and DNA replication were down regulated after berberine treatment. (D)The heat map showed that the differentially expressed genes were down-regulated after the treatment of berberine (only the names of survival related genes were marked on the right). (E)The results showed that berberine significantly affected the survival genes in cell cycle and DNA replication, especially in DNA replication pathway. (F) The image showed that berberine had more significant effect on differential survival related genes in DNA replication pathway. 
A

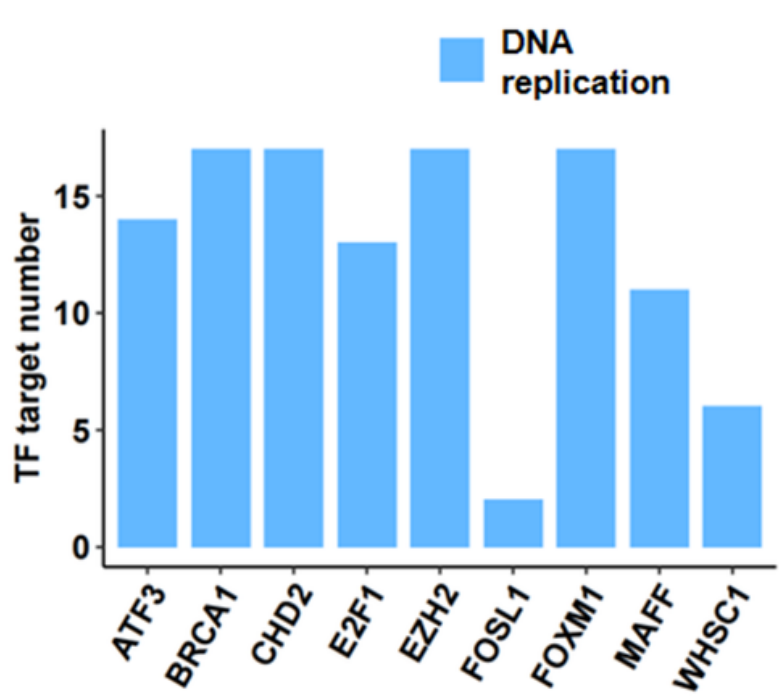

B

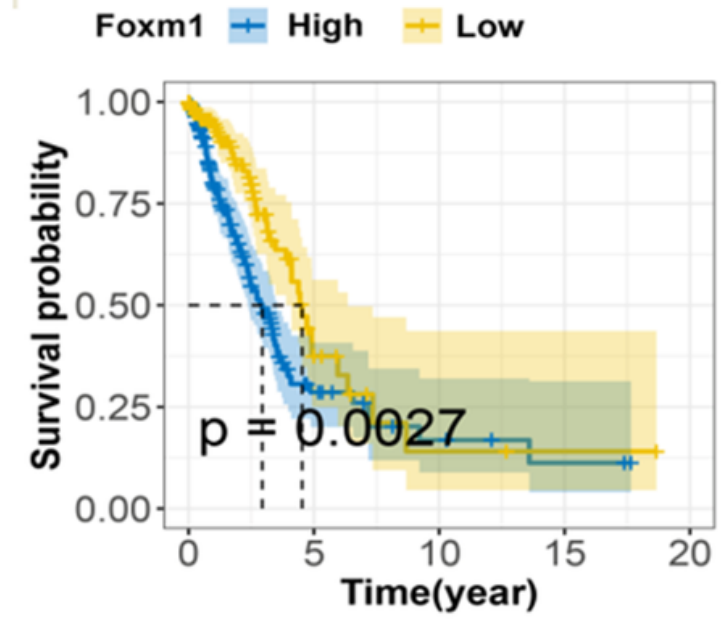

C

A549

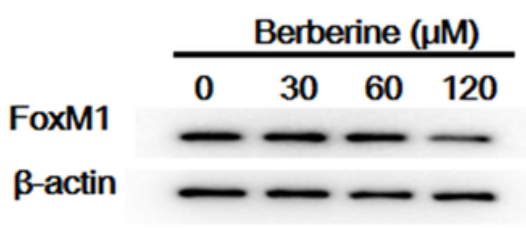

A549

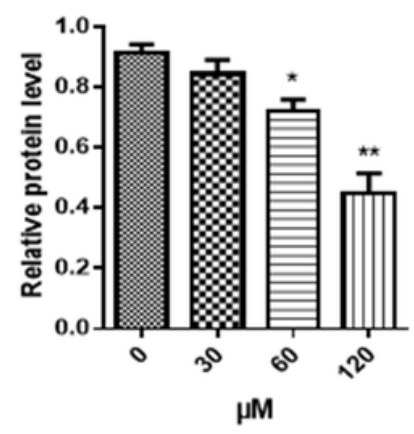

H1299

FoxMl

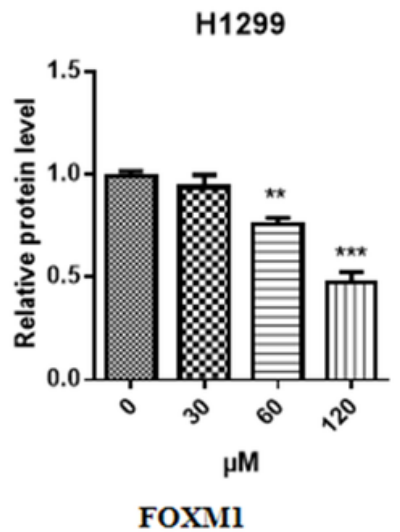

H1299

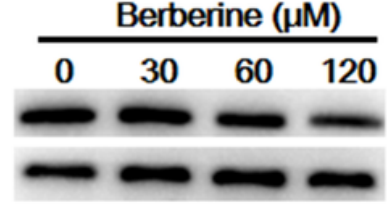

H1975

Berberine $(\mu \mathrm{M})$

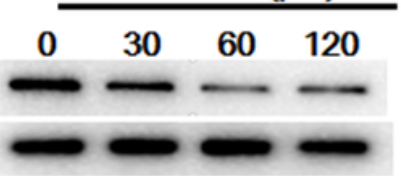

H1975

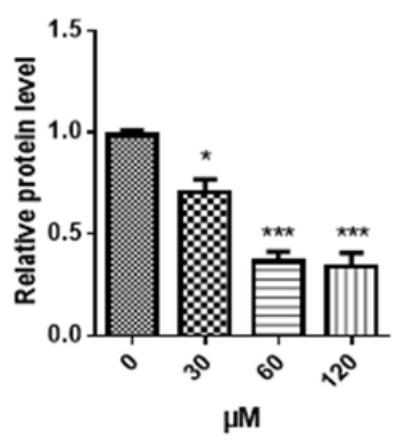

\section{Figure 3}

Berberine down regulated the expression of FoxM1 related to survival. (A)Transcriptional regulation analysis of DNA replication pathway related differential genes showed that the number of target genes regulated by TF or TF cofactors was almost the same except FOSL1. (B)Survival analysis of TFs in TCGA showed only FoxM1 met $\mathrm{P}<0.05$, FoxM1 expression was high and survival was low. (C)Berberine downregulated FOXM1 expression by WB in both A549, H1299 and H1975 cells. (D)Data were pooled from three independent experiments. ${ }^{*}, \mathrm{P}<0.05$, $* \star, P<0.01$, $* \star \star, ~ P<0.001$, compared with the control group. 
A
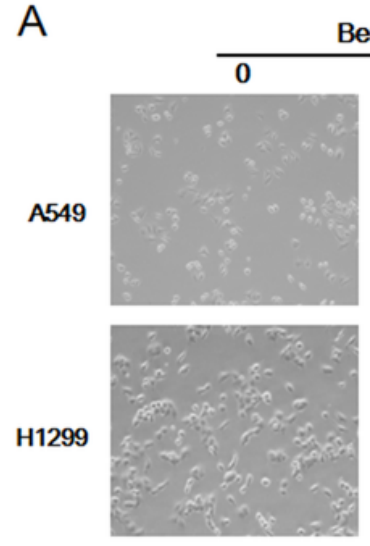

Berberine
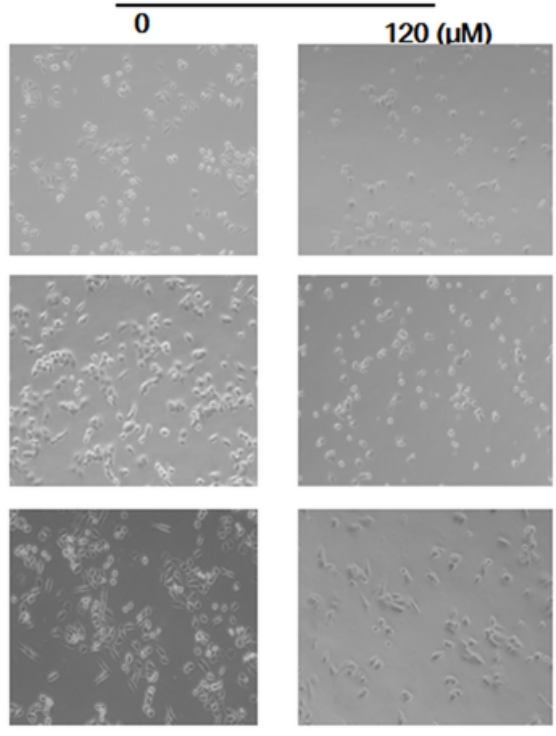

B
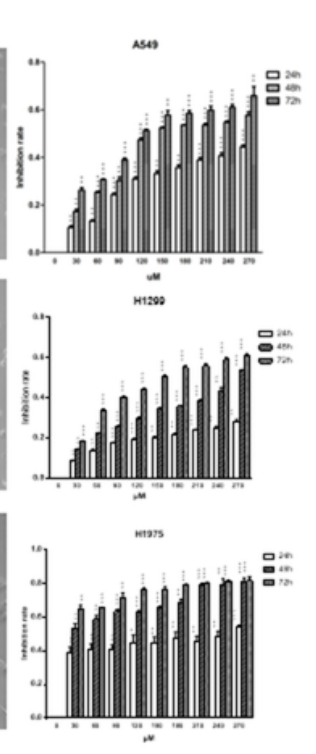

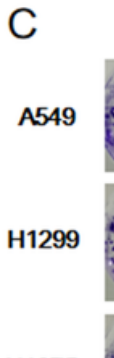

H1975

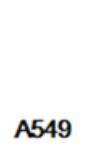

H1299
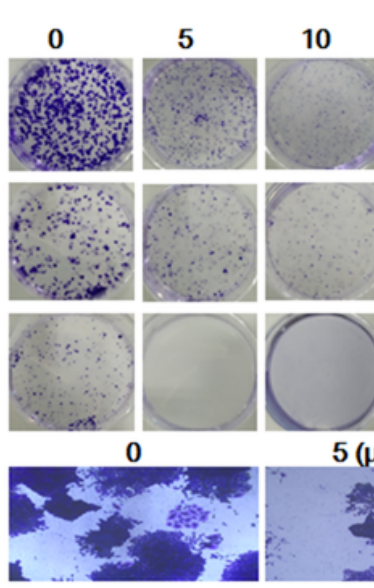

10 $20(\mu \mathrm{M})$
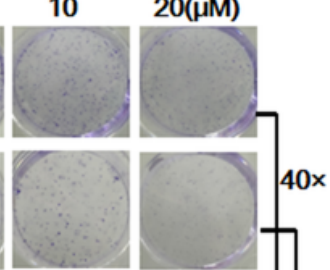

$0 x$
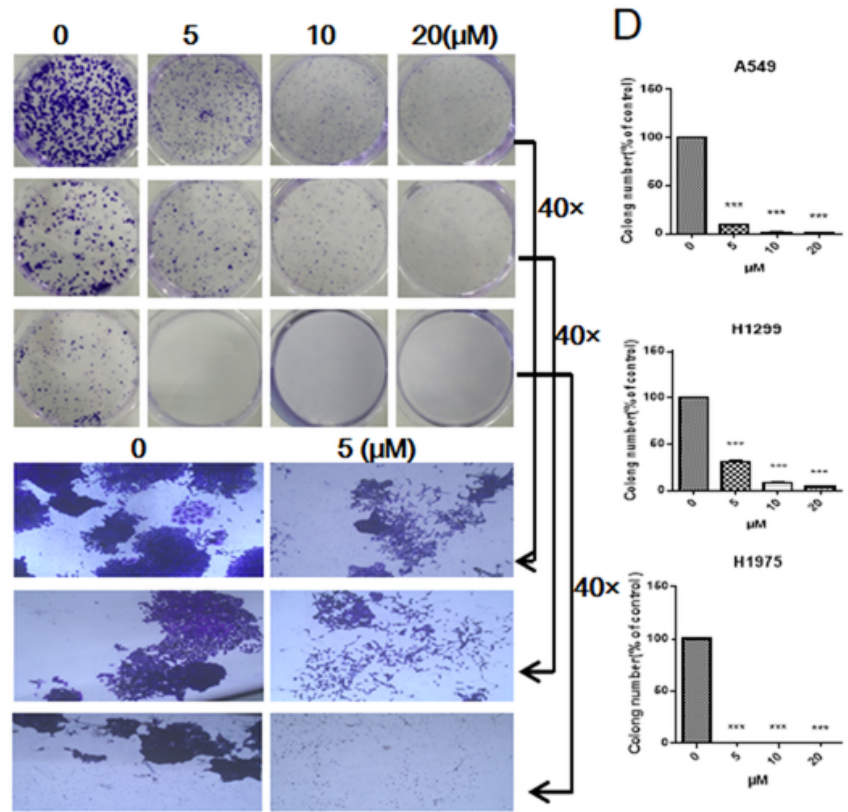

H1299

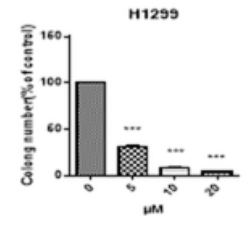

41975

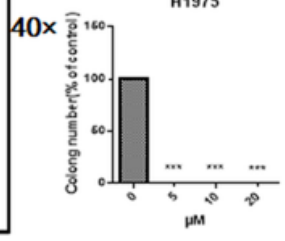

\section{Figure 4}

Berberine inhibited the survival of NSCLC cells. (A) A549, $\mathrm{H} 1299$, and $\mathrm{H} 1975$ cells were treated with berberine $(120 \mu \mathrm{M})$ for $24 \mathrm{~h}$, respectively. Photographs of cell morphology from a representative experiment are shown. (B)A549, $\mathrm{H} 1299$, and $\mathrm{H} 1975$ cells were treated with berberine $(0,30,60,90,120,150,180,210,240,270 \mu \mathrm{M})$ for 24,48 , and $72 \mathrm{~h}$, respectively, and cell viability was analyzed by the MTT assay. (C) A549, H1299, and H1975 cells were treated with berberine $(0,5,10$ and $20 \mu \mathrm{M})$ and allowed to grow for an additional 10 days. Photographs of cell culture dishes from a representative experiment are shown. (B)Display of individual clone sizes $(0$ and5 $\mu \mathrm{M})$. (D) Colony percentages represent the mean \pm SEM from three individual experiments. * $P<0.05, * *, P<0.01, * \star *, P<0.001$, compared with the control group. 
A

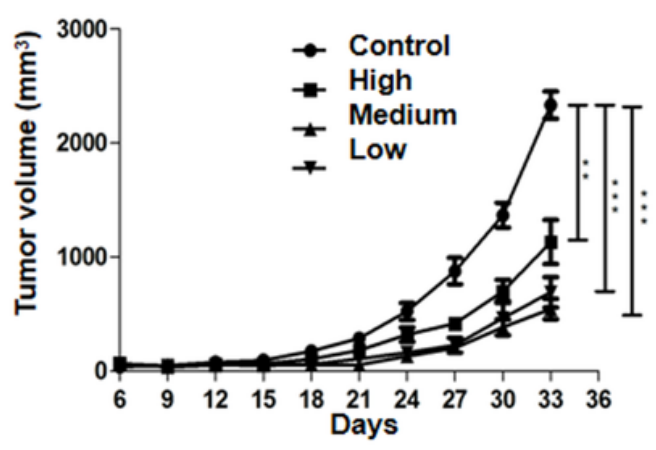

C

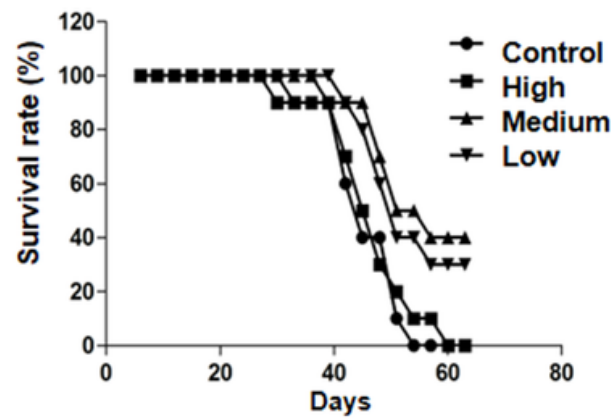

E

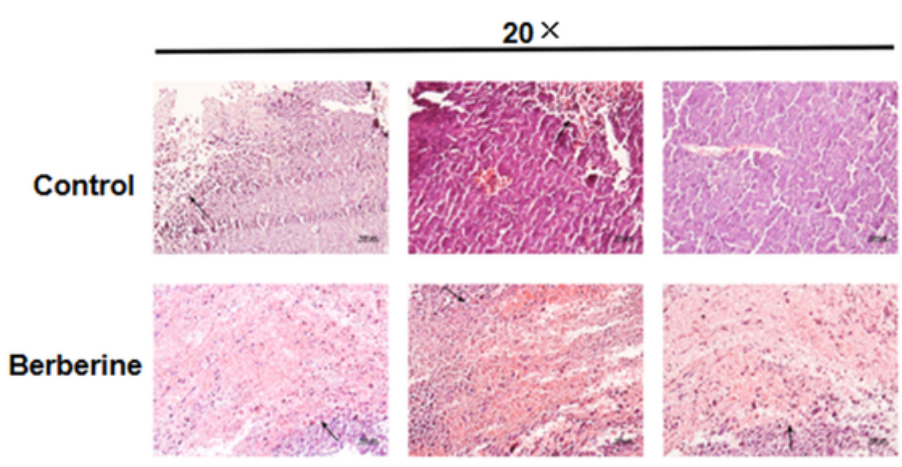

B

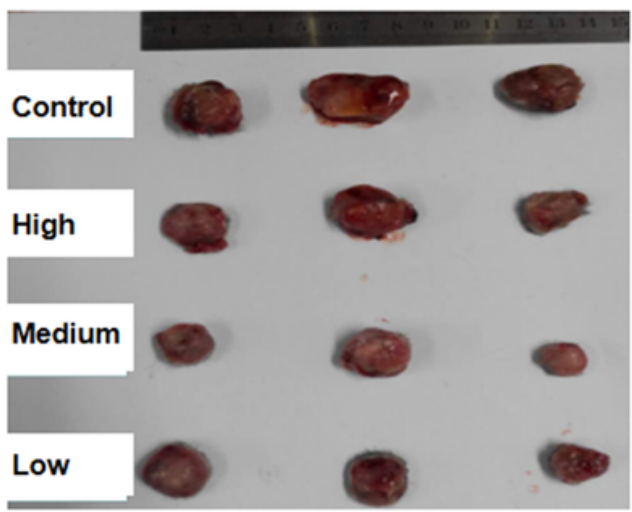

D

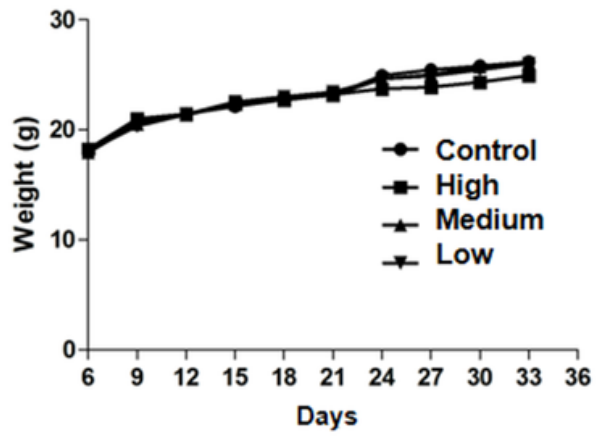

$\mathrm{F}$

ays

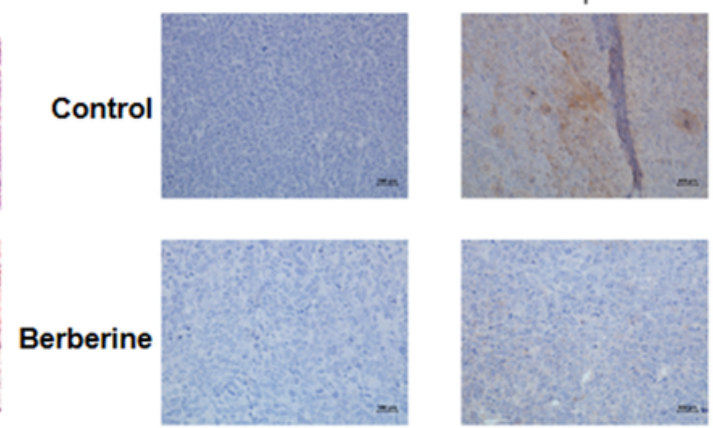

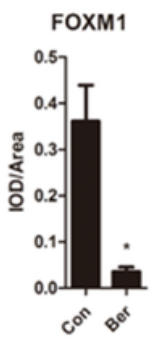

\section{Figure 5}

Berberine inhibited the survival of lung cancer xenografts and down regulated the expression of FoxM1 in vivo. (A)Tumor volume. (B)Tumor photos. (C)Survival. (D)Weight. (E) HE staining of the tumor (The area indicated by the arrow was the tumor necrosis area in mice.) (F) Compared with the blank control group, the expression levels of tumor FOXM1 in mice in the medium concentration berberine group were relatively significantly reduced ("-" refers to the absence of a corresponding antibody, while "+" refers to the corresponding antibody). Data are presented as mean \pm SEM from three independent experiments. *, $\mathrm{P}<0.05, * \star, \mathrm{P}<0.01, * \star \star, \mathrm{P}<0.001$, compared with the control group. 
A
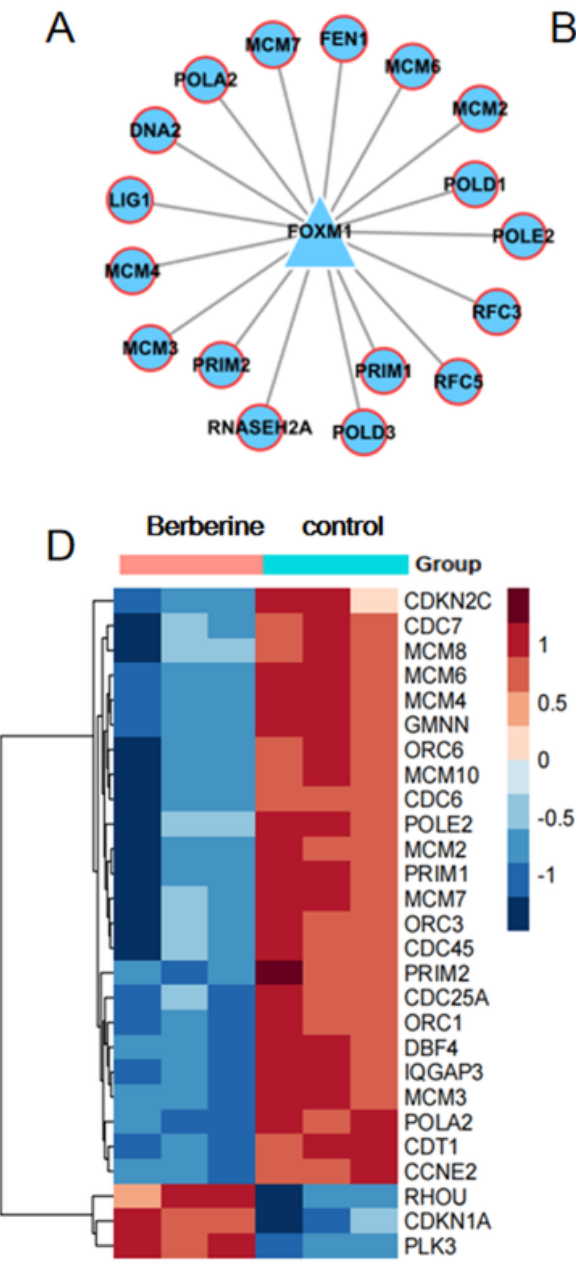

B

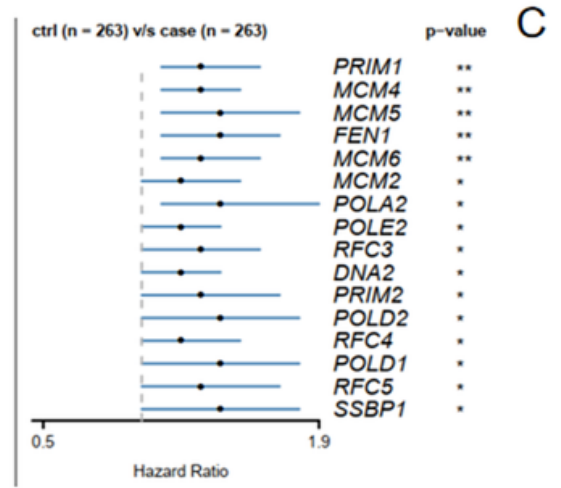

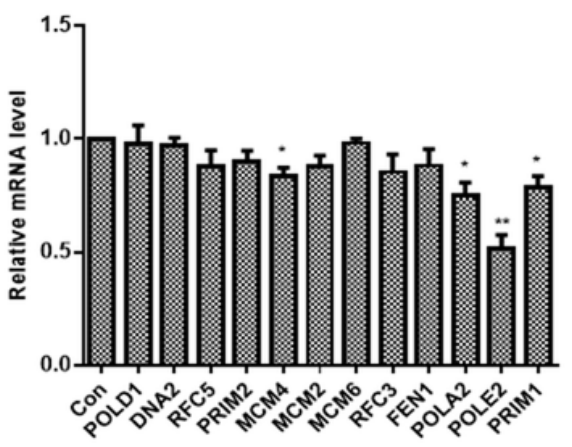

E
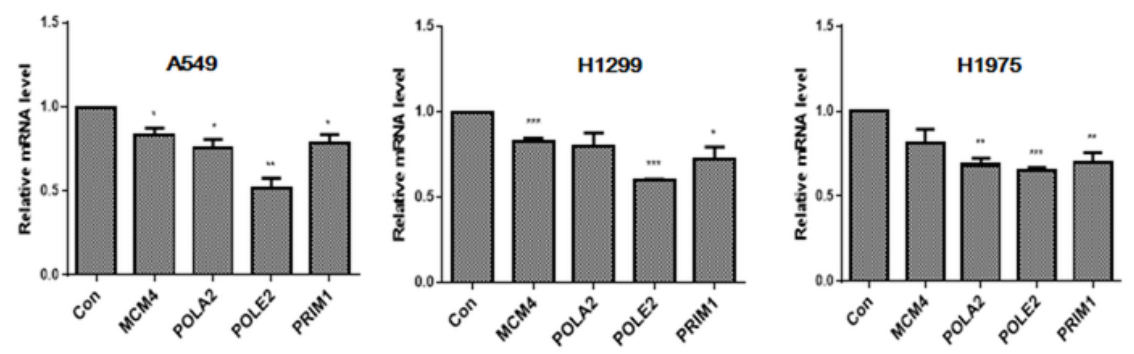

$\mathrm{F}$
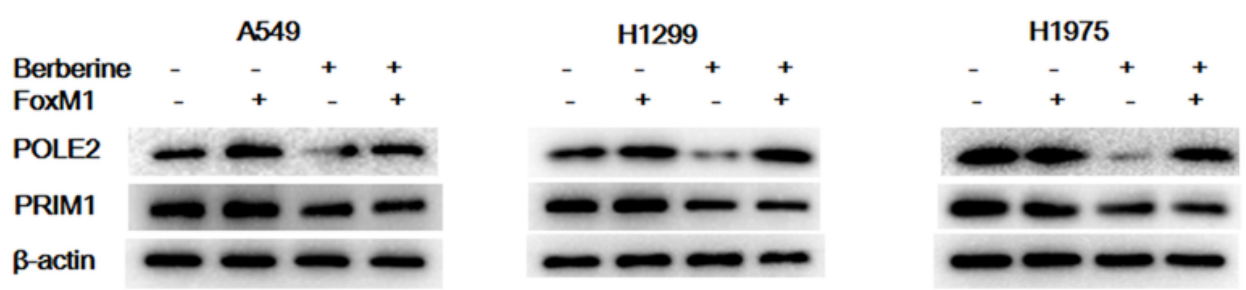

Figure 6

Berberine interfered the expression of survival related genes POLE2 in DNA replication mediated by FoxM1. (A)FoxM1 regulates DNA replication pathway related differential genes. (B)To screen the differential genes which were related to DNA replication pathway in TCGA, and analyze the survival related genes by univariable Cox proportional hazards region analysis. The horizontal axis represents the risk ratio, the middle point represents the value of the risk ratio, and the two ends of the blue line represent the upper and lower limits of the $95 \%$ confidence interval of the risk ratio. (C)We selected common genes both in DNA replication related genes regulated by FoxM1 in A549 cells and DNA replication related survival genes in TCGA, and the expression levels of them in A549 cells were tested by PCR after berberine treatment $(90 \mu \mathrm{M}) .{ }^{*}, \mathrm{P}<0.05, * \star, \mathrm{P}<0.01$, $* \star *, \mathrm{P}<0.001$, compared with the control group. (D)The heat map showed that MCM4, POLA2, POLE2 and PRIM1 were involved in G1/S transition of mitotic cell cycle and DNA replication, while berberine could significantly down regulate their expression, especially on POLE2 and PRIM1. (E)Berberine regulated MCM4, POLA2, POLE2 and PRIM1expression by PCR in both A549, H1299 and H1975 cells. (F)After overexpression of FoxM1 or treatment with berberine, the expression levels of POLE2 and PRIM1 were detected by WB in A549, H1299 and H1975 cells. 


\section{Supplementary Files}

This is a list of supplementary files associated with this preprint. Click to download.

- SupplementaryFigure1.tif

- SupplementaryFigure2.tif 